\title{
EDS and VP-SEM: Practical Considerations and Challenges
}

\author{
Jens Rafaelsen ${ }^{1}$, T. Nylese ${ }^{1}$
}

1. EDAX Inc, A Division of Ametek, Materials Analysis Division, Mahwah, NJ, USA

The variable pressure scanning electron microscope (VP-SEM) has become an invaluable tool for imaging and analyzing non-conductive and un-coated samples that would otherwise charge under high vacuum conditions in the SEM. The presence of a gas in the microscope chamber helps to remove charge buildup on the sample surface but also introduces scattering of the electron beam. This scattering results in a primary un-scattered beam and a beam skirt that can be described as [1]:

$$
r_{s}=\left(\frac{364 Z}{E}\right)\left(\frac{p}{T}\right)^{1 / 2} L^{3 / 2}
$$

where $r_{s}$ is the skirt radius in $\mathrm{m}, Z$ the atomic number of the gas, $E$ the beam energy in $\mathrm{eV}, p$ the pressure in $\mathrm{Pa}, T$ the gas temperature, and $L$ the beam path length in gas in $\mathrm{m}$.

While there is no shortage of articles dealing with electron scattering and energy dispersive spectrometry (EDS) in the VP-SEM [2] [3] [4] [5] and correction methods for quantitative analysis [6] [7] [8], a large group of these articles deal with optimizing variables for the best possible results. This often includes use of gases not readily available to most SEM users (He or $\mathrm{He}-\mathrm{H}_{2} \mathrm{mix}$ ), impractical working distances for EDS due to acceptance angle and shadowing, cumbersome sample preparation and mounting, as well as high acceleration voltages that introduce additional challenges for light element detection and quantification.

In this work we will take a look at the effect of variable pressure under normal working conditions for EDS, which is typically around $10 \mathrm{~mm}$ working distance (WD), $40 \mathrm{~Pa}$ pressure and relatively high beam current. An example is shown in Figures Figure 1-Figure 4 where an Ir wire of $250 \mu \mathrm{m}$ diameter is embedded in Ag epoxy in a steel matrix. At high vacuum a spectrum from the center of the wire only shows Ir with traces of $\mathrm{C}$ and $\mathrm{O}$, but even at $10 \mathrm{~Pa}$ an $\mathrm{Ag}$ signal is apparent and at $40 \mathrm{~Pa}$ the Fe peak from the matrix starts to show. The shortest distance to the matrix is roughly $750 \mu \mathrm{m}$ from the wire indicating that the beam skirt is in excess of this distance which is significantly higher than what would be suggested by using the WD for the gas path length in Equation (1). Since the gas path length is highly dependent on the column design of the specific microscope and generally not the same as the WD we will look at two different microscopes and estimate the beam skirt and EDS signal contribution from the skirt through high resolution line scans. We will also look at the relative contributions depending on distance from the analysis point as well as the background elevation that follows from EDS analysis under VP conditions.

References:

[1] G. Danilatos, Adv. Electronics Electron Phys. 71 (1988), p. 109.

[2] D. E. Newbury, Journal of Research of the National Institute of Standards and Technology 107 (2002), p. 567.

[3] L. Khouchaf, Microscopy Research 1 (2013), p. 29.

[4] J. Rattenberger, J et al, Scanning 31 (2009), p. 107. 
[5] D. E. Newbury and N. W. M. Ritchie, Microsc. Microanal. 23 (2017), p. 1090.

[6] E. Doehne, Scanning 19 (1997), p. 75.

[7] J. F. Mansfield, Microchimica Acta 132 (2000), p. 137.

[8] R. Gauvin, Scanning 21 (1999), p. 388.

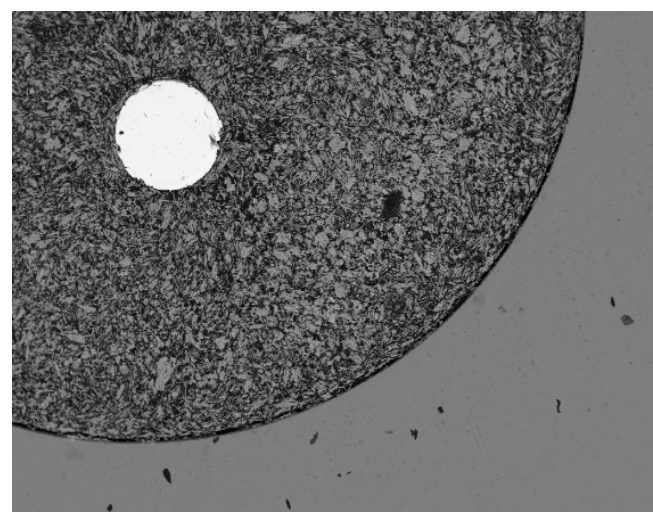

Figure 1. Backscatter image of sample showing Ir wire embedded in Ag epoxy surrounded by a steel matrix.

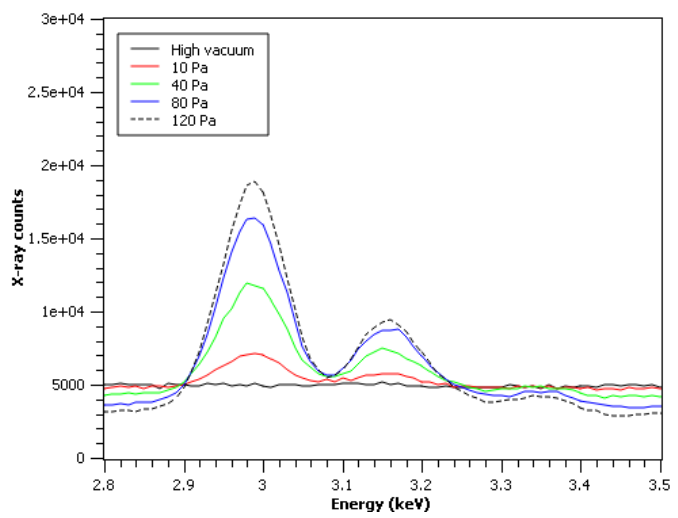

Figure 3. EDS spectra from center of Ir wire showing Ag contribution increasing with pressure.

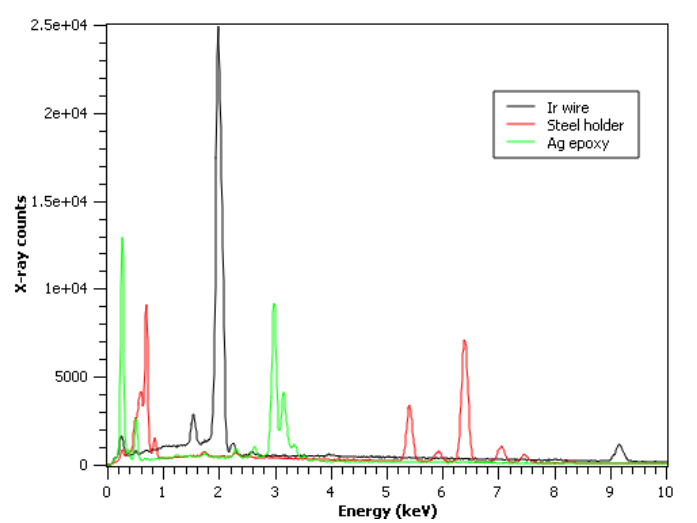

Figure 2. EDS spectra of Ir wire, Ag epoxy, and steel matrix.

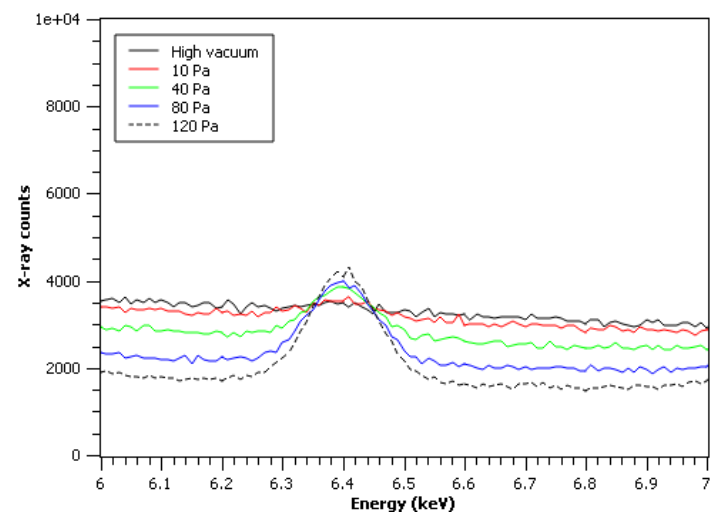

Figure 4. EDS spectra from center of Ir wire showing Fe contribution increasing with pressure. 